\title{
Plantas usadas como medicinais no município de Ipê, RS, Brasil
}

\author{
Ritter, M.R. ${ }^{{ }^{*}}$; Sobierajski, G.R. ${ }^{3}$; Schenkel, E.P. ${ }^{2}$; Mentz, L.A. ${ }^{1}$ \\ ${ }^{1}$ Departamentoto. Botânica, Instituto de Biociências, Universidade Federal do Rio Grande do Sul, \\ Porto Alegre, RS; \\ ${ }^{2}$ Departamento de Ciências Farmacêuticas, Centro de Ciências da Saúde, Universidade Federal de \\ Santa Catarina, Florianópolis, SC; \\ ${ }^{3}$ Bióloga, Bolsista de Extensão PROREXT-UFRGS.
}

\begin{abstract}
Recebido para publicação em: 27/03/2002 Aceito para publicação em: 26/08/2002.

RESUMO: Foi realizado um levantamento das plantas medicinais utilizadas pela população do município de Ipê, RS, Brasil, visando uma investigação da medicina tradicional, melhoramento e racionalização das práticas medicinais populares, em um projeto envolvendo voluntários da comunidade. Foram entrevistadas 114 pessoas, que mencionaram 252 plantas, das quais foram identificadas 105 espécies, agrupadas em 48 famílias. As plantas coletadas e identificadas foram analisadas, de acordo com dados químicos, farmacológicos e/ou toxicológicos encontrados na literatura científica. Os resultados obtidos mostram que a população deste município faz uso de plantas que, ainda, não foram alvo de pesquisa química, farmacológica e/ou toxicológica e, também, que a mesma faz uso de onze espécies, que são reconhecidamente, responsáveis por efeitos colaterais indesejados, ou ainda, que são tóxicas.
\end{abstract}

Unitermos: plantas medicinais; medicina popular; Rio Grande do Sul.

ABSTRACT: Medicinal plants used by people from Ipê, Rio Grande do Sul State, Brazil. Medicinal plants used by Brazilian people from Ipê city, in Rio Grande do Sul State, were the subject of a survey realized in order to investigate the traditional medicine in this comunity. The project involved 114 individuals who cited 252 medicinal plants. From these plants, 105 species were identified and classified into 48 botanical families. Assessments of all known plants were based on chemical, pharmacological and toxicological data searched in scientific literature. The results show that many of these plants were not yet chemically and/or pharmacologically and/or toxicologically studied, and eleven of them were related to be responsible for side effects or even toxicity in the consulted scientific literature.

Key words: medicinal plants; folk medicine; Rio Grande do Sul State.

\section{INTRODUÇÃO}

As plantas que nos rodeiam, como qualquer ser vivo, produzem substâncias químicas, que podem atuar beneficamente sobre outros organismos ou agirem de forma tóxica. Portanto, para que o homem possa fazer uso medicinal de uma espécie, com segurança, é necessário que a mesma seja estudada sob o ponto de vista químico, farmacológico e toxicológico. Como estes aspectos nem sempre são totalmente avaliados, principalmente, para plantas nativas brasileiras, outros critérios deveriam ser considerados para que elas pudessem ser consideradas como 
medicinais. Dentre esses, estão o uso tradicional e amplo, durante décadas ou algumas centenas de anos, e a coincidência de usos entre diferentes populações. Nesses casos, determinadas plantas poderiam ser recomendadas no tratamento de doenças autolimitadas, da mesma forma como é admitido o uso de medicamentos de venda livre, sem prescrição médica, descartando-se, no entanto, o uso em doenças infecciosas e distúrbios metabólicos, entre outras afecções, que requerem acompanhamento médico. Para doenças destas categorias, o uso de plantas não encontra justificativa, sendo necessária uma avaliação clínica e, muitas vezes, até internação hospitalar.

Vários levantamentos etnobotânicos foram realizados em diferentes municípios do Rio Grande do Sul, tais como Kubo (1997) (Coronel Bicaco), Garlet (2000) (Cruz Alta), Marodin (2000) (Dom Pedro de Alcântara) e Possamai (2000) (Mariana Pimentel), evidenciando a diversidade de espécies vegetais utilizadas como medicinais, pelos diversos grupos étnicos, que deram origem a população atual do Estado e proporcionando subsídios para um maior conhecimento da flora medicinal regional.

Este é um trabalho de investigação sobre a medicina tradicional de uma parcela da população do município de Ipê, RS, com vistas ao melhoramento e racionalização das práticas medicinais populares, fundamentadas no uso de plantas referidas como seguras e ressaltando aquelas reconhecidas como tóxicas, com o objetivo de promover a integração entre o conhecimento original da comunidade e aquele advindo dos meios acadêmicos.

\section{MATERIAIS E MÉTODOS}

O projeto foi realizado no município de Ipê, RS, envolvendo voluntários da comunidade (professores das redes estadual e municipal de ensino e agentes comunitários), os quais foram responsáveis pelas pesquisas de campo, realizada com 114 moradores voluntários, em suas residências, localizadas nos diferentes distritos. Esses últimos foram escolhidos aleatoriamente, sendo 82 mulheres e 32 homens. O modelo de questionário utilizado encontra-se na página seguinte.

O município de Ipê tem uma população de 5.456 habitantes (IBGE, 2000), com uma taxa de alfabetização de 90,5 \%, e compõe-se de três distritos (Sede, Vila Segredo e Vila São Paulo). Possui área de $742 \mathrm{~km}^{2}$, distribuída entre serras e campos, e está situado no nordeste do Estado, na região fisiográfica denominada Campos de Cima da Serra.

Para o desenvolvimento deste trabalho foram realizadas as seguintes etapas:

a) obtenção de dados sobre o município através da EMATER//pê e Prefeitura Municipal;

b) treinamento de doze voluntários pela EMATER/lpê, responsáveis pela realização das entrevistas e coleta das plantas utilizadas como medicinais pela comunidade;

c) identificação das espécies coletadas e incorporação das exsicatas ao Herbário ICN da UFRGS (foram consideradas todas as plantas citadas nas entrevistas e que puderam ser identificadas até espécie);

d) análise das informações obtidas nas entrevistas e tabulação dos dados de todas as coletas de cada espécie, para comparação das informações;

e) revisão da literatura científica para cada espécie, obtendo-se informações sobre seus usos medicinais, suas composições químicas e suas atividades farmacológicas e toxicológicas, através de levantamentos em bases de dados disponíveis, dissertações de Mestrado realizadas no Estado do RS e livros específicos desta área. 
Modelo da ficha de entrevista

Entrevistador: Entrevista ${ }^{\circ}$ :

Entrevistado: Data:

Distrito: Município:

Nomes populares:

Hábito de crescimento: erva ( ) arbusto ( ) árvore ( ) trepadeira ( ) outro:

Ambiente de coleta: mato ( ) campo ( ) capoeira ( ) terra abandonada ( ) beira de estrada ( ) cultivada( ) outro:

Flores (cor): tamanho da planta:

Frutos:

Parte(s) da planta utilizada:

Usos:

Modo de preparo: chá (água)（） maceração ( ) infusão ( ) decocção ( ） chá(leite) ( ) Alcoolatura: cachaça ( ) vinho ( ) outro:

Xarope ( ) garrafada ( ) compressa ( ) ungüento ( )

Banhos ( ) cataplasma ( ) pomada ( ) inalação ( )

Observações adicionais:

Modo de administração/dosagem:

Procedimento para colheita ou preparo:

Época do ano para a colheita da planta para uso:

Costuma guardar a planta seca para utilização posterior?

Conhece contra-indicação para o uso da planta?

Conhece efeito indesejável que aparece com o uso da planta?

Identificação botânica:

Família:

Determinador: Data: 


\section{RESULTADOS E DISCUSSÃO}

Foram realizadas entrevistas com 114 pessoas, que mencionaram 252 plantas. Essas plantas, após a identificação, resultaram em 105 táxons identificados até espécie e três identificados apenas até gênero (somando-se, então, 108 táxons), agrupados em 48 famílias. Para 15 dos 105 táxons mencionados, não foram encontradas referências de uso popular na literatura consultada, sendo os mesmos excluídos da Tabela 1, que refere, então, somente 90 táxons. Os nomes e os usos populares mencionados neste trabalho (Tabela 1) são os indicados pela comunidade, os quais foram obtidos através das entrevistas. Aquelas plantas que não puderam ser identificadas até espécie, por falta de coleta de partes reprodutivas, foram desconsideradas pela inexistência de outras informações: Begonia sp., begônia-do-mato (Begoniaceae), Rumex sp., língua-de-vaca (Polygonaceae) e Sisyrinchium sp., pêlo-de-porco (Iridaceae). Das 48 famílias identificadas, 33 estão representadas por apenas uma espécie, mostrando uma diversidade interessante, fato também observado em outros levantamentos no Estado (KUBO, 1997; GARLET, 2000; MARODIN, 2000). Em relação ao número de espécies por família, a mais representativa foi Asteraceae, com 24 táxons, seguida de Lamiaceae, com 10 táxons, fato também verificado por Garlet (2000), Kubo, (1997), Marodin, (2000) e Possamai (2000). A seguir, são apresentados e discutidos os riscos de utilização dessas plantas.

\section{- Plantas de uso considerado como seguro}

Algumas plantas são de amplo uso popular, cuja tradição remonta pelo menos há séculos, como é o caso da camomila [Chamomilla recutita (L.) Rauschert] e funcho (Foeniculum vulgare Mill.). A Agência Nacional de Vigilância Sanitária, através da Resolução RDC no 17, de 24 de fevereiro de 2000, permite o registro e, portanto, a produção e comércio, de medicamentos fitoterápicos tradicionais, elaborados a partir de plantas medicinais de uso baseado na tradição popular, cuja eficácia foi validada em trabalhos científicos e sem toxicidade conhecida. Portanto, plantas como a alcachofra (Cynara scolymus L.), a camomila, o funcho e a malva (Malva sylvestris L.) são consideradas seguras. Torna-se necessário, no entanto, chamar a atenção de que plantas como o funcho e a camomila, apesar do uso tradicional e da confirmação científica de suas atividades farmacológicas, não podem ser consideradas como isentas de efeitos colaterais. Para o funcho já foram relatadas dermatites de contato em humanos (LEUNG, 1980), sendo que o óleo volátil obtido dos frutos pode causar convulsões epileptiformes (BURKHARD et al., 1999) e depressão do sistema nervoso central (GIRRE, 1985; WENIGER e ROBINEAU, 1988). Para a camomila, também, já foram relatadas dermatites de contato em seres humanos, desenvolvidas pelo contato repetido com a planta (SIMÕES et al., 1986, PAULSEN e ANDERSEN, 1993).

Plantas utilizadas como antidiarréicas, como a goiaba-do-mato [Acca sellowiana (Berg) Burret], o araçá (Psidium cattleyanum Sabine) e a pitangueira (Eugenia uniflora L.), não foram alvo de investigação profunda, mas têm seu uso justificado devido à presença de taninos, que são reconhecidamente antidiarréicos. No entanto, os usuários devem estar conscientes dos problemas causados pela desidratação comum nos casos de diarréia, e diferenciá-los daqueles oriundos de diarréias infecciosas, que exigem cuidados médicos imediatos.

\section{- Plantas de toxicidade estabelecida}

As plantas com toxicidade reconhecida, cujo uso deve ser desaconselhado, como a arruda, babosa (uso interno), codina, confrei (uso interno), figueira (uso externo), mamona, 
melão-de-são-caetano, quebra-pedra, além dos problemas advindos do uso crônico da losna, são discutidas a seguir.

A arruda (Ruta graveolens L.) é uma planta que contém substâncias tóxicas, como a metilnonilcetona, que excita a motilidade do útero, sendo responsável por abortos, além de substâncias fotossensibilizantes (furanocumarinas, como o bergapteno), causadoras de lesões e queimaduras na pele e mucosas quando expostas ao sol (TYLER, 1987; DUKE, 1989; SOUSA et al., 1991; PARFITT, 1999; WESSNER et al., 1999; MENGUE et al., 2001).

A babosa (Aloe arborescens Mill.), devido à presença de antraquinonas, de conhecida ação laxante, não deve ser indicada para uso interno, já que as referidas substâncias podem causar dores abdominais e irritações no intestino. Em gestantes, por estimular contrações da musculatura lisa uterina, pode provocar aborto (BELEW, 1999). Derivados antraquinônicos são excretados no leite, devendo ser evitados por mulheres que estão amamentando (PARFITT, 1999).

A codina (Chelidonium majus L.), conhecida em outras regiões do Estado como celidônia, iodo ou iodina, é uma planta muito próxima à papoula (Papaver somniferum L.), e como essa, produz alcalóides extremamente tóxicos. Seu uso interno deve ser desaconselhado, pois pode provocar estomatites e gastroenterites (DEBELMAS e DELAVEAU, 1978; GIRRE, 1980 e 1985; WENDELBERGER, 1981; DUKE, 1989), braquicardia, alterações na pressão sangüínea, paralisia e espasmos musculares (DUKE, 1989), além de induzir casos de hepatite em humanos (BENNINGER et al., 1999). O uso externo também deve ser evitado, já que o látex da planta é irritante e vesicante, causando dermatites de contato e, quando em contato com os olhos, lesões sérias (MARTINS et al., 1994).

O confrei (Symphytum officinale L.) foi indicado, pelas pessoas entrevistadas, para o tratamento da asma, diabete, hepatite, gastrite e reumatismo, como regulador da pressão e, em uso externo, como cicatrizante. Esta planta contém alcalóides pirrolizidínicos, de ação carcinogênica e hepatotóxica, além de serem responsáveis por aborto, teratogênese e mutagênese (GIRRE, 1980; SIMÕES et al., 1986; DUKE, 1989; SOUSA et al., 1991; BELEW, 1999; PRAKASH et al., 1999; MENGUE et al., 2001). Seu uso interno foi proibido no Brasil através da Portaria n.10 da Secretaria Nacional da Vigilância Sanitária, de 30 de janeiro de 1992. Apenas em uso externo, como cicatrizante, o uso desta espécie é justificado, sendo permitida a produção de medicamentos fitoterápicos de uso tópico através da Resolução RDC n.17, de 24 de fevereiro de 2000.

O figo ou figueira (Ficus carica L.) possui um látex cáustico, rico em furanocumarinas fotossensibilizantes, as quais causam danos sérios quando em contato com a pele e mucosas expostas à luz solar (GIRRE, 1980; LEMBO et al., 1985; SCHVARTSMAN, 1992; MARTINS et al., 1994; SCHENKEL et al., 2000).

As sementes da mamona (Ricinus communis L.) contêm um óleo com pronunciada ação purgante, irritante para a membrana intestinal, com concomitante perda de proteínas e potássio (PARIS e MOYSE, 1967). Os sintomas da intoxicação são náuseas, vômitos, cólicas, diarréias, suores frios, pulso rápido, hipotensão, colapso, tremores e estupor. Casos de intoxicação em humanos estão bem documentados na literatura mundial (WEDIN et al., 1986; FERNANDO e FERNANDO, 1990; JASPEREN-SCHIB et al., 1996). Algumas sementes podem provocar a morte de uma criança (GIRRE, 1980; WENIGER e ROBINEAU, 1988; SCHENKEL et al., 2000) e a ingestão de 15 sementes, a morte de um adulto (GIRRE, 1980).

O melão-de-são-caetano (Momordica charantia L.) não deve ser utilizado internamente, devido à reconhecida toxicidade de suas sementes, que provocaram aborto, em camundongos (CHAN et al., 1984; MENGUE et al., 2001). Os usos relatados pelos informantes entrevistados não correspondem àqueles consagrados pela literatura, como as ações emenagoga, purgativa e anti-helmíntica (DUKE, 1989; MARTINS et al., 1994). 
O quebra-pedra utilizado pelos entrevistados corresponde à Euphorbia serpens H.B.K. As espécies do gênero Euphorbia contêm um látex extremamente cáustico, o qual pode causar lesões na pele (SCHMIDT e EVANS, 1980; SANTUCCl et al., 1985), nos olhos (SCOTT e KARP, 1996) e, quando ingerido, na mucosa gastrointestinal; pode ainda provocar lesões renais (SCHENKEL et al., 2000). Os usos referidos para esta planta têm relação com o seu nome popular, quebra-pedra, isto é, como possível litolítico. Este mesmo uso é referido, na literatura, para outra espécie (Phyllanthus niruri L.), para a qual existem alguns estudos não conclusivos, relacionados com a atividade mencionada.

A losna (Artemisia absinthium L.) é uma planta amplamente difundida para uso em problemas digestivos. É necessário chamar a atenção para a presença de um componente neurotóxico, a $\alpha$-tujona, cujo mecanismo de intoxicação já foi elucidado (HÖLD et al., 2000). A tujona é responsável por vômitos, tremores e convulsões (PARFITT, 1999). A losna, quando ingerida, em grandes quantidades, pode provocar crises epileptiformes e até mesmo aborto (SIMÕES et al., 1986; DUKE, 1989). Por isto, seu uso continuado deve ser evitado (GIRRE, 1980 e 1985; LEUNG, 1980; TYLER, 1987; DUKE, 1989; MARTINS et al., 1994). O absintismo, isto é, o uso continuado de bebidas alcoólicas produzidas com losna, é um distúrbio que se manifesta através de convulsões e perturbações da consciência, com degeneração irreversível do sistema nervoso central (MENGUE et al., 2001). Com base nestas informações, a losna é uma planta que deve ser utilizada com muita precaução. Mesmo assim, alguns autores consideram o seu uso não prudente, já que existem outras plantas mais seguras (ROBBERS e TYLER, 1999).

\section{- Plantas desconhecidas quanto ao risco/benefício}

Não foram encontradas referências científicas, que justifiquem o uso das seguintes espécies, nas afecções mencionadas nas entrevistas: Amaranthus blintun L., nome popular não mencionado (Amaranthaceae); Blepharocalyx salicifolius (H.B.K.) Berg, multa (Myrtaceae); Bauhinia microstachya (Raddi) Macbride, cipó-pata-de-vaca (Caesalpiniaceae); Carya illinoensis (Wangh.) Koch, nogueira (Juglandaceae); Chaptalia exscapa (Pers.) Baker, fumo-mulum, fumo-verde (Asteraceae); Chrysanthemum myconis L., calêndula (Asteraceae); Cyphomandra corymbiflora Sendtn., malvão-do-mato, malvão, eslavação (Solanaceae); Dicksonia sellowiana Hook., xaxim (Dicksoniaceae); Digitaria ciliaris (Retz.) Koel., capim-calção (Poaceae); Hypoestes sanguinolenta Hook., anador (Acanthaceae); Juncus capillaceus Lam., capim-pêlo-de-porco (Juncaceae); Microgramma squamulosa (Kaulf.) De la Sota, cipó-barbado (Polypodiaceae); Paspalum distichum L., gramínea (Poaceae); Poiretia tetraphylla (Poir.) Burkart, erva-de-touro (Fabaceae) e Pterocaulon interruptum DC., doce-amargo (Asteraceae).

\section{- Denominações de plantas}

Para algumas espécies, os nomes populares consagrados, fornecidos pelos informantes, não corresponderam aos referidos na literatura. Assim, Apium leptophyllum (Pers.) F.Muell. (Apiaceae), conhecido como aipo-chimarrão e aipo-bravo, para a população do município corresponde a anis ou erva-doce, e Chelidonium majus L., denominada na literatura por celidônia, iodo ou iodina, é conhecida no município por codina.

É interessante destacar que nomes comerciais de medicamentos, como anador, infalivina, melhoral e penicilina, foram informados como nomes populares de plantas. Em diversos levantamentos realizados em outros municípios do RS, tais nomes foram mencionados para diferentes espécies, conforme explicitado a seguir.

O nome anador, para Hypoestes sanguinolenta Hook. (Acanthaceae), espécie originária de Madagascar, foi, neste trabalho, mencionado para o tratamento de dores em geral e gripe. No

Rev. Bras. Farmacogn., v. 12, n. 2, jul.-dez. 2002. 
município de Cruz Alta, o nome anador é utilizado para Achillea millefolium L. (Asteraceae), também no tratamento da gripe, entre outros usos (GARLET, 2000). Já no município de Dom Pedro de Alcântara, o nome anador refere-se a Alternanthera ficoidea (L.) R.Brown (Amaranthaceae), indicada no tratamento de dores em geral (MARODIN, 2000). É interessante observar que no município de Coronel Bicaco, outra espécie do mesmo gênero é conhecida como anador (Alternathera reineckii Briq.), indicada no tratamento da dor de cabeça (KUBO, 1997).

O nome melhoral, para Salvia microphylla H.B.K. (Lamiaceae), espécie originária do México, foi mencionado no tratamento da dor de cabeça, gripe, resfriado, qualquer dor, má circulação do sangue e palpitação do coração. No município de Cruz Alta, o nome melhoral corresponde a essa mesma espécie, tendo sido também indicada em febres, dor de cabeça e gripe (GARLET, 2000). No município de Coronel Bicaco, outra espécie de Lamiaceae é conhecida como melhoral, Melissa officinalis L., referida para o tratamento de dores agudas (KUBO, 1997). No município de Dom Pedro de Alcântara, o nome melhoral não foi mencionado no levantamento realizado por MARODIN (2000).

Estes dados confirmam que a indicação de nomes de medicamentos para plantas vem sendo observada, nos últimos anos (KUBO, 1997; GARLET, 2000; MARODIN, 2000), mostrando a necessidade que as pessoas têm em buscar substitutivos mais baratos nos cuidados à saúde, fenômeno que pode aumentar os riscos de acidentes ou intoxicações na população.

\section{CONCLUSÃO}

Os resultados obtidos mostraram que esta população faz uso de um grande número de plantas, que ainda não foram alvo de investigações científicas. Para apenas 11 das 105 espécies identificadas foram encontradas referências que indicam segurança e eficácia para alguns dos usos indicados naquela população: Achyrocline satureioides (Lam.) DC., Calendula officinalis L. (uso externo), Chamomilla recutita (L.) Rauscher, Cynara scolymus L., Foeniculum vulgare Mill., Lactuca sativa L., Malva sylvestris L., Maytenus ilicifolia Mart. ex Reissek, Rosmarinus officinalis L., Salvia officinalis L. e Symphytum officinale L. (uso externo). Observou-se, também, que a mesma faz uso de, pelo menos, 11 espécies que podem ser classificadas como responsáveis por efeitos colaterais não desejados e/ou efeitos tóxicos. Por isso, pode-se questionar a segurança e a eficácia dos métodos de atenção à saúde utilizados por esta comunidade, evidenciando-se a necessidade de maior envolvimento dos agentes de saúde e de um processo educativo continuado.

Tabela 1. Plantas referidas nas entrevistas e identificadas até espécie, com os nomes populares e usos informados pelos entrevistados, no município de Ipê, RS. *

\begin{tabular}{|c|c|c|}
\hline $\begin{array}{c}\text { Nome científico } \\
\text { Família }\end{array}$ & Nomes populares & Usos populares referidos nas entrevistas \\
\hline $\begin{array}{l}\text { Acca sellowiana (Berg) Burret } \\
\text { Myrtaceae }\end{array}$ & goiaba-do-mato & diarréia, baixar a pressão \\
\hline $\begin{array}{l}\text { Achillea millefolium L. } \\
\text { Asteraceae }\end{array}$ & $\begin{array}{l}\text { ponta-alívio, } \\
\text { mil-em-ramas }\end{array}$ & $\begin{array}{l}\text { gripes, dores em geral, febres, catarro, pontadas (pneumo- } \\
\text { nia), nervos, infecção no intestino, rins, bexiga, queimadu- } \\
\text { ras, hemorróidas }\end{array}$ \\
\hline $\begin{array}{l}\text { Achyrocline satureioides (Lam.) DC. } \\
\text { Asteraceae }\end{array}$ & $\begin{array}{l}\text { marcela, } \\
\text { macela }\end{array}$ & $\begin{array}{l}\text { dor de cabeça, dor de estômago, para fazer a digestão, } \\
\text { para o fígado, diarréia, tosses, gripe }\end{array}$ \\
\hline $\begin{array}{l}\text { Achyrocline vauthieriana DC. } \\
\text { Asteraceae }\end{array}$ & marcela & dores no estômago e má digestão \\
\hline Adiantum raddianum Presl. & avenca & catarro pulmonar, ronquidão e tosse \\
\hline
\end{tabular}

* A terminologia utilizada para referir os usos e a grafia dos nomes populares correspondem aos mencionados nas entrevistas. 


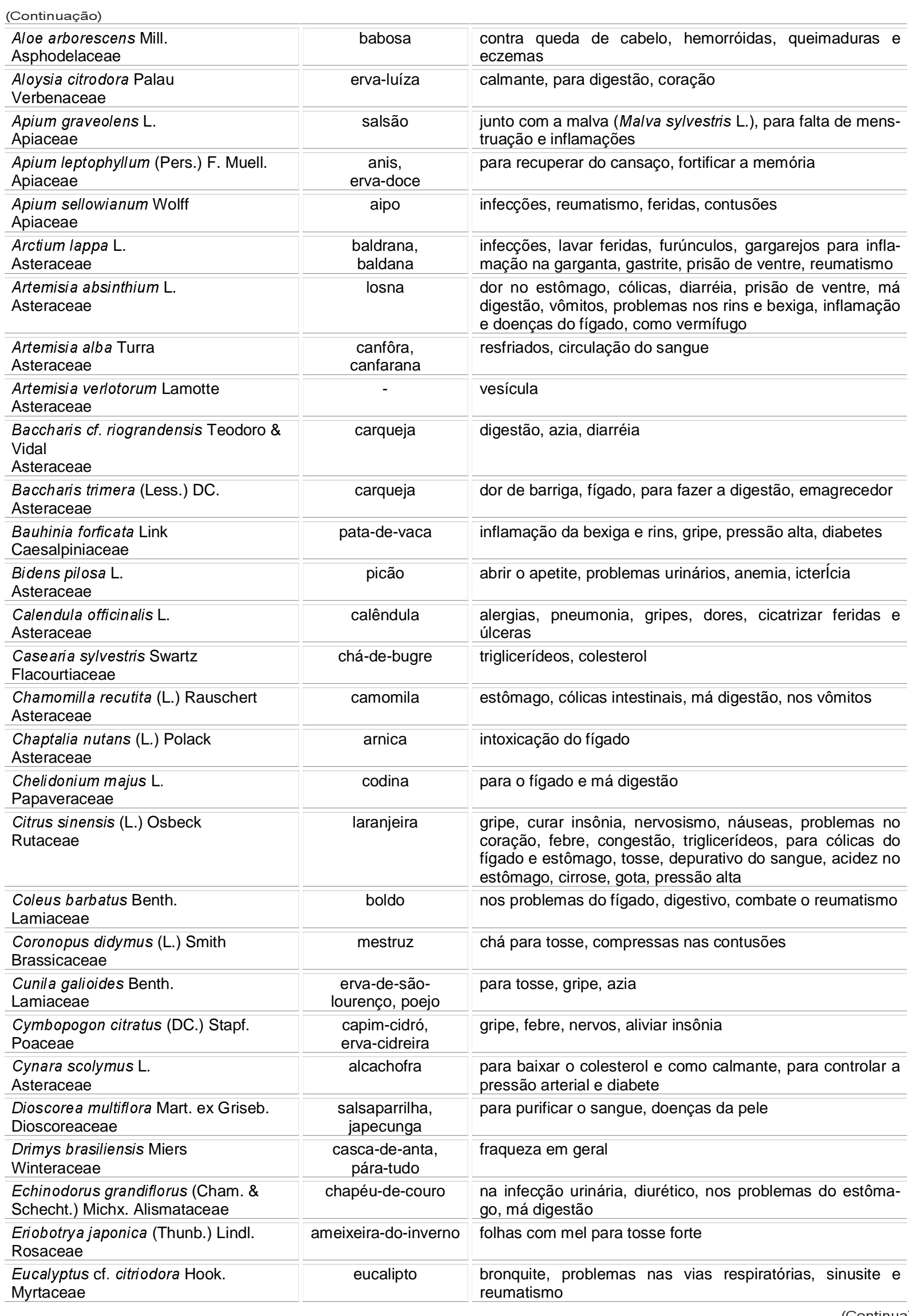


(Continuação)

Eugenia uniflora L.

Myrtaceae

Euphorbia serpens H.B.K.

Euphorbiaceae

Ficus carica L.

Moraceae

Foeniculum vulgare Mill.

Apiaceae

Fragaria vesca $\mathrm{L}$.

Rosaceae

Gochnatia polymorpha (Less.) Cabrera

Asteraceae

Heimia salicifolia (H.B.K.) Link

Lythraceae

Lactuca sativa $\mathrm{L}$.

Asteraceae

Lantana megapotamica (S-

preng.)Troncoso

Verbenaceae

Laurus nobilis L.

Lauraceae

Leonurus sibiricus L.

Lamiaceae

Lippia alba (Mill.) N.E.Br.

Verbenaceae

Luehea divaricata Mart.

Tiliaceae

Malva sylvestris $\mathrm{L}$.

Malvaceae

Maytenus ilicifolia Mart. ex Reissek Celastraceae

Marrubium vulgare $\mathrm{L}$.

Lamiaceae

Mentha spicata L.

Lamiaceae

Mikania laevigata Sch.Bip. ex Baker

Asteraceae

Momordica charantia L.

Cucurbitaceae

Muehlenbeckia sagittifolia (Ort.)

Meissn.

Polygonaceae

Myrocarpus frondosus Freire Alemão

Fabaceae

Nasturtium officinale R. Brown

Brassicaceae

Ocimum selloi Benth.

Lamiaceae

Origanum cf. majorana L.

Lamiaceae

Olea europaea $L$.

Oleaceae

Parapiptadenia rigida (Benth.) Brenner

Mimosaceae

Passiflora edulis Sims

Passifloraceae

Petiveria alliacea $\mathrm{L}$.

pitangueira
quebra-pedra
figueira
funcho
erva-doce
moranguinho

cambará-do-mato

sarandi
alface

sabiá-do-mato

lauro, laureiro-dos-poetas erva-do-santo-filho, macaé, erva-raposa

salva

açoita-cavalo,

mutamba-preta

malva

cancorosa, espinheira-santa gervão

hortelã-pimenta

guaco

balsami, melão-desão-caetano

salsaparrilha

gabriuva

agrião

alfavaca, manjericão-de-folha-larga, remédio-de-vaqueiro manjerona

oliva
angico
maracujá
guiné

diarréia, normalizar a pressão, cólicas, baixar o colesterol

pedras nos rins

o leite para queimar as verrugas

para aumentar o leite quando estiver amamentando, inflamação intestinal, prisão de ventre, nos gases e cólicas abdominais, diurético

na hepatite, controlar a pressão arterial e a bronquite asmática

gripe, como expectorante, pneumonia

para lavar feridas

calmante

dores no estômago e fígado

para reumatismo (só as folhas), úlceras (frutos e folhas com mel), ausência da menstruação

contra infecção, inflamação e dor no estômago

inflamações na garganta, picada de insetos, gripe

reumatismo e diarréia

coceira, feridas, dor de ouvido; inflamação nos dentes, garganta e gengiva; aliviar tosses, bronquite e asma; na inflamação do estômago, intestino, bexiga e útero; prisão de ventre; junto com o salsão (Apium graveolens L.) para falta de menstruação e infecções

estômago, úlceras, azia

má digestão e dor de cabeça

nervos, insônia e dores de cabeça

bronquite, tosse, gripe, reumatismo, febre, gota

para prevenir a gripe

para feridas, frieiras, picadas de insetos, depurativo do sangue, gota, reumatismo

tosse, gripe

expectorante e para esquecimento

rins, ardor ao urinar, gripe, resfriado, tosse, febre

febre

diurético

para tratar e curar a asma

asma, coqueluche, diarréia, dor de cabeça e crises nervosas

inflamações, dor de dente e cabeça, sobre feridas como 
(Continuação)

Petroselinum crispum (Mill.) A.W.Hill

Apiaceae

Phyllanthus niruri L.

Euphorbiaceae

Plantago australis Lam.

Plantaginaceae

Pluchea sagittalis (Lam.) Cabrera

Asteraceae

Polygonum punctatum Elliot

Polygonaceae

Psidium cattleyanum Sabine

Myrtaceae

Punica granatum L.

Punicaceae

Richardia brasiliensis Gómes

Rubiaceae

Ricinus communis L.

Euphorbiaceae

Rosmarinus officinalis L.

Lamiaceae

\section{Rubus erythrocladus Mart.}

Rosaceae

Ruta graveolens $\mathrm{L}$.

Rutaceae

Salvia officinalis $\mathrm{L}$.

Lamiaceae

Salvia microphylla H.B.K.

Lamiaceae

Sechium edule Swartz

Cucurbitaceae

Sida rhombifolia L.

Malvaceae

Smilax cognata Kunth

Smilacaceae

Symphytum officinale L.

Boraginaceae

Tagetes minuta $\mathrm{L}$.

Asteraceae

Tanacetum vulgare $\mathrm{L}$.

Asteraceae

Taraxacum officinale Weber ex Wig-

gers

Asteraceae

Tetragonia expansa Murray

Aizoaceae

Tropaeolum pentaphyllum Lam.

Tropaeolaceae

Verbena cf. intermedia Gill. et Hook.

Verbenaceae

Verbena montevidensis Spreng.

Verbenaceae

Viola odorata L.

Violaceae

Vitex megapotamica (Spreng.)

Moldenke

Verbenaceae

Zea mays L.

Poaceae salsão

quebra-pedra

tanchagem,

tansagem

arnica

erva-de-bicho

araçá

romã

amendoim-do-mato

mamona, carrapatei-

ro, palma-cristi

alecrim,

alecrim-do-jardim

mana-branca

arruda

sálvia,

sabiá

melhoral,

melissa

chuchu

guaxuma

salsaparrilha

confrei

canego, cravo-de-defunto catinga-de-mulata

dente-de-leão, radichi-de-mato,

espinafre

men

gervão-da-folhabranca, fel-da-terra gervão

violeta

tarumã, taromão

pendão, flores-de-milho na infecção e inflamação, bexiga, baixar a pressão, hepatite, má digestão diurético, dor nos rins, bexiga, urina trancada, pedra nos rins

úlceras, ardor no estômago, impurezas do sangue, inflamações e feridas

má digestão, fígado, dor de dente, dor de cabeça, pressão alta, passar em machucados, batidas e cortes

baixar a febre, nos problemas circulatórios, na disenteria, como vermífugo, hemorróidas

diarréia

disenteria

para dor de barriga, cólica provocada por vermes

como vermífugo

as folhas secas para cicatrizar feridas, o chá para lavar feridas, palpitação no coração, fazer a digestão, problemas com o fígado, fortificante

colesterol, purificar o sangue

cólicas menstruais

estômago, congestão, vômito, gripe, inflamações, catarro pulmonar e feridas

dor de cabeça, gripe, resfriado, qualquer dor, má circulação do sangue, palpitação do coração

para pressão alta

queda de cabelo

para circulação do sangue

regular a pressão, cicatrizante, asma, diabete, hepatite, gastrite e reumatismo

para curar a asma

para o fígado, asma, gota e reumatismo

para limpar o sangue, para falta de apetite, diurético, laxante e cólicas do fígado

contra anemia e prisão de ventre

para diabetes (flor) e gripe (raiz)

pontada, fígado, abrir o apetite

fígado (dor, intoxicação)

asma, coqueluche, gripe, bronquite, tosse, sarampo, inflamação na garganta

para purificar o sangue e para baixar os triglicerídeos e o colesterol

para os rins, como diurético

Rev. Bras. Farmacogn., v. 12, n. 2, jul.-dez. 2002. 


\section{AGRADECIMENTOS}

Os autores agradecem à Ari H. Uriartt (Eng. Agrônomo - EMATER/RS) por propiciar a realização deste trabalho, à Ana L.C.B. Meirelles (Eng. Agrônoma CAE/lpê/RS) e à Eneiva L.C. Balancelli (Extensionista EMATER/RS) pela disponibilidade em participarem e coordenarem o trabalho de campo; à Lenise Guerra e Regina Soares (Prefeitura Municipal de Ipê) pela colaboração; e aos professores e agentes comunitários do Município de Ipê, responsáveis pelas entrevistas (Alzira Andreatta, Carmen Grazziotin, Eremy Bortolotto, Hilário Mattana, Iriete Zanotto, Lisiane Paim Majero, Lúcia Girotto, Maurílio Candiago, Renita Zampieri, Teresinha Alessi). O agradecimento é extensivo aos botânicos, que identificaram várias das espécies: Bruno Edgar Irgang, Marcos Guerra Sobral, Nelson Ivo Matzenbacher, Rosana Moreno Senna, Rumi Kubo, Sérgio Augusto de Loreto Bordignon e Thais Scotti do Canto-Dorow.

\section{REFERÊNCIAS BIBLIOGRÁFICAS}

BELEW, C. Herbs and the childbearing woman - guidelines for midwives. Journal of Nurse Midwifery, v.44, n.3, p.231-252, 1999.

BENNINGER, J.; SCHNEIDER, H.T.; SCHUPPAN, D.; KIRCHNER, T.; HAHN, E.G. Acute hepatitis induced by greater celandine (Chelidonium majus). Gastroenterology, v.117, n.5, p.1234-1237, 1999.

BURKHARD, P.R.; BIRKHARD, K.; HAENGGELI, C.A.; LANDIS, T. Plant-induced seizures: reappearance of an old problem. Journal of Neurology, v.246, n.8, p.667-670, 1999.

CHAN, W.Y.; TAM, P.P.; YEUNG, H.W. The termination of early pregnancy in the mouse by beta-momorcharin. Contraception, v.29, n.1, p.91-100, 1984.

DEBELMAS, A.M.; DELAVEAU, P. Guide des plantes dangereuses. Paris: Maloine, 1978.

DUKE, J.A. Handbook of medicinal herbs. Boca Raton: CRC, 1989.

FERNANDO, R.; FERNANDO, D.N. Poisoning with plants and mushrooms in Sri Lanka: a retrospective hospital based study. Veterinary and Human Toxicology, v.32, n.6, p.579-581, 1990.

GARLET, T.M.B. Levantamento das plantas medicinais utilizadas no município de Cruz Alta, RS, Brasil. Porto Alegre, 220p. Dissertação (Mestrado) - Programa de Pós-Graduação em Botânica, Universidade Federal do Rio Grande do Sul, 2000.

GIRRE, L. Connaître et reconnaître les plantes médicinales. Rennes: Ouest France, 1980.

GIRRE, L. Nouveau guide des vieux remèdes naturels. Rennes: Ouest France, 1985.

HÖLD, K.M.; SIRISOMA, N.S.; IKEDA, T.; NARAHASHI, T.; CASIDA, J.E. $\alpha$-thujone (the active component of absinthe): $\gamma$-Aminobutyric acid type A receptor modulation and metabolic detoxification. Proceedings of National Academy of Sciences, v.97, n.8, p.3826-3831, 2000.

JASPEREN-SCHIB, R.; GUIRGUIS-OESCHGER, M.; GOSSWEILER, B.; MEIER-ABT, P.J. Wichtige Pflanzenvergiftungen in der Schweiz 1966-1994. Eine Fallanalyse aus dem Schweizerischen Toxikologischen Informationszentrum. Schweizerische Medizinische Wochenschrift, v. 126, n.25, p.1085-1098, 1996.

KUBO,R.R. Levantamento das plantas de uso medicinal em Coronel Bicaco, RS. Porto Alegre, 163p. Dissertação (Mestrado) - Programa de Pós-Graduação em Botânica, Universidade Federal do Rio Grande do Sul, 1997.

LEMBO, G.; LO PRESTI, M.; BALATO, N. 1985. Phytophotodermatitis due to Ficus carica. Photo-Dermatology, V.2, n.2, p.119-120, 1985.

LEUNG, A.Y. Encyclopedia of common natural ingredients used in food, drugs and cosmetics. New York: John Wiley, 1980.

MARODIN, S.M. Plantas utilizadas como medicinais no município de Dom Pedro de Alcântara, Rio Grande do Sul. Porto Alegre, 413p. Dissertação (Mestrado) - Programa de Pós-Graduação em Botânica, Universidade Federal do Rio Grande do Sul, 2000. 
MARTINS, E.R.; CASTRO, D.M.; CASTELLANI, D.C.; DIAS, J.E. Plantas medicinais. Viçosa: UFV - Imprensa Universitária, 1994.

MENGUE, S.S.; MENTZ, L.A.; SCHENKEL, E.P. Uso de plantas medicinais na gravidez. In: SANSEVERINO, M.T.V.; SPRITZER, D.T.; SCHÜLLER-FACCINI, L.(org.) Manual de teratogênese. Porto Alegre: Editora da Universidade/UFRGS, p. 423-450, 2001.

PARFITT, H. (ed.) Martindale: the complete drug reference. 32 ${ }^{\text {nd }}$ ed. London: Pharmaceutical Press, 1999. PARIS, R.R.; MOYSE, H. Précis de Matière Médicale. Paris: Masson, 1967. v.2.

PAULSEN, E.; ANDERSEN, K.E. Compositae dermatitis in a Danish Dermatology Department in 1 year. 2. Clinical-features in patients with Compositae contact allergy. Contact Dermatitis, v.29, n.4, p.195-201, 1993.

POSSAMAI, R.M. Levantamento etnobotânico das plantas de uso medicinal em Mariana Pimentel, RS. Porto Alegre, 108p. Dissertação (Mestrado) - Programa de Pós-Graduação em Botânica, Universidade Federal do Rio Grande do Sul, 2000.

PRAKASH, A.S.; PEREIRA, T.N.; REILLY, P.E.B.; SEAWRIGHT, A.A. Pyrrolizidine alkaloids in human diet. Mutation Research, v.443, p.53-67, 1999.

ROBBERS, J.E.; TYLER, V.E. Tyler's Herbs of choice: the therapeutic use of phytomedicinals. New York: Haworth Herbal, 1999.

SANTUCCI, B.; PICARDO, M.; CRISTAUDO, A. Contact dermatitis from Euphorbia pulcherrima. Contact Dermatitis, v.12, n.5, p.285-286, 1985.

SCHENKEL, E..P.; ZANNIN, M.; MENTZ, L.A.; BORDIGNON, S.A.L.; IRGANG, B. Plantas tóxicas. In: SIMÕES, C.M.O.; SCHENKEL, E.P.; GOSMANN, G.; MELLO, J.C.P.; MENTZ, L.A.; PETROVICK, P.R. (Org.). Farmacognosia: da planta ao medicamento. Porto Alegre: Editora da Universidade/UFRGS; Florianópolis: Editora da UFSC, p.755-788, 2000.

SCHMIDT, R.J.; EVANS, F.J. Skin irritants of the sun spurge (Euphorbia helioscopia L.). Contact Dermatitis, v.6, n.3, p.204-210, 1980.

SCHVARTSMAN, S. Plantas venenosas e animais peçonhentos. São Paulo: Sarvier, 1992.

SCOTT, I.U.; KARP, C.L. Euphorbia sap keratopathy: four cases and a possible pathogenic mechanism. British Journal of Ophtalmology, v.80, p.823-826, 1996.

SIMÕES, C.M.O.; MENTZ, L.A.; SCHENKEL, E.P. ; IRGANG, B. E.; STEHMANN, J. R. Plantas da medicina popular no Rio Grande do Sul. Porto Alegre: Editora da Universidade/UFRGS, 1986.

SOUZA, M.P.; MATOS, M.E.O.; MATOS, F.J.A.; MACHADO, M.I.L.; CRAVEIRO, A.A. Constituintes químicos ativos de plantas medicinais brasileiras. Fortaleza: UFC/Laboratório de Produtos Naturais, 1991.

TYLER, V.E. The new honest herbal. Philadelphia: George F. Stickley Company, 1987.

WEDIN, G.P.; NEAL, J.S.; EVERSON, G.W.; KRENZELOK, E.P. Castor bean poisoning. American Journal of Emergency Medicine, v.4, n.3, p.259-261, 1986.

WENDELBERGER, E. Pequeña guia de las plantas medicinales: determinación y uso de las principales especies mediante fotografias en color. Barcelona: Omega, 1981.

WENIGER, B.; ROBINEAU, L. Elementos para una farmacopea Caribeña. La Habana: ENDA-CARIBE, 1988. (Seminário, 3, TRAMIL).

WESSNER, D.; HOFMANN, H.; RING, J. Phytophotodermatitis due to Ruta graveolens applied as protection against evil spells. Contact Dermatitis, v.41, n.4, p.232, 1999.

* Autor para correspondência

Profa. Mara Rejane Ritter

Depto. de Botânica - Instituto de Biociências

Universidade Federal do Rio Grande do Sul

Av. Bento Gonçalves, 9500

Prédio 43433 - Campus do Vale

91501-970 - Porto Alegre - RS

E-mail: mrritter@terra.com.br 